PROCEEDINGS OF THE

AMERICAN MATHEMATICAL SOCIETY

Volume 138, Number 11, November 2010, Pages 3933-3943

S 0002-9939(2010)10319-2

Article electronically published on July 13, 2010

\title{
STABILITY CRITERION FOR CONVOLUTION-DOMINATED INFINITE MATRICES
}

\author{
QIYU SUN \\ (Communicated by Marius Junge)
}

\begin{abstract}
Let $\ell^{p}$ be the space of all $p$-summable sequences on $\mathbb{Z}$. An infinite matrix is said to have $\ell^{p}$-stability if it is bounded and has bounded inverse on $\ell^{p}$. In this paper, a practical criterion is established for the $\ell^{p}$-stability of convolution-dominated infinite matrices.
\end{abstract}

\section{INTRODUCTION}

Let $\mathcal{C}$ be the Gohberg-Baskakov-Sjöstrand class of infinite matrices $A:=$ $\left(a\left(j, j^{\prime}\right)\right)_{j, j^{\prime} \in \mathbb{Z}}$ with

$$
\|A\|_{\mathcal{C}}=\sum_{k \in \mathbb{Z}^{j}} \sup _{j-j^{\prime}=k}\left|a\left(j, j^{\prime}\right)\right|<\infty .
$$

Let $\ell^{p}:=\ell^{p}(\mathbb{Z})$ be the set of all $p$-summable sequences on $\mathbb{Z}$ with the standard norm $\|\cdot\|_{p}$. An infinite matrix $A:=\left(a\left(j, j^{\prime}\right)\right)_{j, j^{\prime} \in \mathbb{Z}} \in \mathcal{C}$ defines a bounded linear operator on $\ell^{p}, 1 \leq p \leq \infty$, in the sense that

$$
A c=\left(\sum_{j^{\prime} \in \mathbb{Z}} a\left(j, j^{\prime}\right) c\left(j^{\prime}\right)\right)_{j \in \mathbb{Z}},
$$

where $c=(c(j))_{j \in \mathbb{Z}} \in \ell^{p}$. Given a summable sequence $h=(h(j))_{j \in \mathbb{Z}} \in \ell^{1}$, define the convolution operator $C_{h}$ on $\ell^{p}, 1 \leq p \leq \infty$, by

$$
C_{h}: \ell^{p} \ni(b(j))_{j \in \mathbb{Z}} \longmapsto\left(\sum_{k \in \mathbb{Z}} h(j-k) b(k)\right)_{j \in \mathbb{Z}} \in \ell^{p} .
$$

Observe that the linear operator associated with an infinite matrix $A \in \mathcal{C}$ is dominated by a convolution operator in the sense that

$$
|(A c)(j)| \leq\left(C_{h}|c|\right)(j):=\sum_{j^{\prime} \in \mathbb{Z}} h\left(j-j^{\prime}\right)\left|c\left(j^{\prime}\right)\right|, \quad j \in \mathbb{Z},
$$

for any sequence $c=(c(j))_{j \in \mathbb{Z}} \in \ell^{p}, 1 \leq p \leq \infty$, where $|c|=(|c(j)|)_{j \in \mathbb{Z}}$ and the sequence $\left(\sup _{j-j^{\prime}=k}\left|a\left(j, j^{\prime}\right)\right|\right)_{k \in \mathbb{Z}}$ can be chosen to be the sequence $h=(h(j))_{j \in \mathbb{Z}}$ in (1.3). So infinite matrices in the set $\mathcal{C}$ are said to be convolution-dominated.

Convolution-dominated infinite matrices were introduced by Gohberg, Kaashoek, and Woerdeman [12] as a generalization of Toeplitz matrices. They showed that the class $\mathcal{C}$ equipped with the standard matrix multiplication and the above norm $\|\cdot\|_{\mathcal{C}}$

Received by the editors October 14, 2008 and, in revised form, November 30, 2009.

2010 Mathematics Subject Classification. Primary 47B35; Secondary 40E05, 65F05, 42C40, 47G30, 94A20.

(C)2010 American Mathematical Society Reverts to public domain 28 years from publication 
is an inverse-closed Banach subalgebra of $\mathcal{B}\left(\ell^{p}\right)$ for $p=2$. Here $\mathcal{B}\left(\ell^{p}\right), 1 \leq p \leq \infty$, is the space of all bounded linear operators on $\ell^{p}$ with the standard operator norm, and a subalgebra $\mathcal{A}$ of a Banach algebra $\mathcal{B}$ is said to be inverse-closed if when an operator $T \in \mathcal{A}$ has an inverse $T^{-1}$ in $\mathcal{B}$, then $T^{-1} \in \mathcal{A}([7,11,21])$. The inverse-closed property for convolution-dominated infinite matrices was rediscovered by Sjöstrand [25] with a completely different proof and an application to a deep theorem about pseudodifferential operators. Recently Shin and Sun 23 generalized Gohberg, Kaashoek and Woerdeman's result and proved that the class $\mathcal{C}$ is an inverse-closed Banach subalgebra of $\mathcal{B}\left(\ell^{p}\right)$ for any $1 \leq p \leq \infty$. The readers may refer to $[5,10,20,23,25,27$ and the references therein for related results and various generalizations on the inverse-closed property for convolution-dominated infinite matrices.

Convolution-dominated infinite matrices arise and have been used in the study of spline approximation ([8, 9]), wavelets and affine frames ([6, 18,), Gabor frames and non-uniform sampling ([3, 14, 15, 26]), and pseudo-differential operators ([13, 16, 24, 25 and the references therein). Examples of convolution-dominated infinite matrices include the infinite matrix $\left(a\left(j-j^{\prime}\right)\right)_{j, j^{\prime} \in \mathbb{Z}}$ associated with convolution operators and the infinite matrix $\left(a\left(j-j^{\prime}\right) e^{-2 \pi \sqrt{-1} \theta j^{\prime}\left(j-j^{\prime}\right)}\right)_{i, j \in \mathbb{Z}}$ associated with twisted convolution operators, where $\theta \in \mathbb{R}$ and the sequence $a=(a(j))_{j \in \mathbb{Z}}$ satisfies $\sum_{j \in \mathbb{Z}}|a(j)|<\infty([1,14,19,27,29])$.

A convolution-dominated infinite matrix $A$ is said to have $\ell^{p}$-stability if there are two positive constants $C_{1}$ and $C_{2}$ such that

$$
C_{1}\|c\|_{p} \leq\|A c\|_{p} \leq C_{2}\|c\|_{p} \text { for all } c \in \ell^{p} .
$$

The $\ell^{p}$-stability is one of basic assumptions for infinite matrices arising in the study of spline approximation, Gabor time-frequency analysis, nonuniform sampling, and algebra of pseudo-differential operators; see [1, 3, 6, 8, 9, 10, 14, 15, 16, 18, 19, 23, [24, 25, 26, 27, 29] and the references therein. Practical criteria for the $\ell^{p}$-stability of a convolution-dominated infinite matrix will play important roles in the further study of those topics.

However, up to the knowledge of the author, little is known about practical criteria for the $\ell^{p}$-stability of an infinite matrix. For an infinite matrix $A=$ $\left(a\left(j-j^{\prime}\right)\right)_{j, j^{\prime} \in \mathbb{Z}}$ associated with convolution operators, there is a very useful criterion for its $\ell^{p}$-stability. It states that $A$ has $\ell^{p}$-stability if and only if the Fourier series $\hat{a}(\xi):=\sum_{j \in \mathbb{Z}} a(j) e^{-i j \xi}$ of the generating sequence $a=(a(j))_{j \in \mathbb{Z}} \in \ell^{1}$ does not vanish on the real line, i.e.,

$$
\hat{a}(\xi) \neq 0 \text { for all } \xi \in \mathbb{R} .
$$

Applying this criterion for the $\ell^{p}$-stability, one concludes that the spectrum $\sigma_{p}\left(C_{a}\right)$ of the convolution operator $C_{a}$ as an operator on $\ell^{p}$ is independent of $1 \leq p \leq \infty$, i.e.,

$$
\sigma_{p}\left(C_{a}\right)=\sigma_{q}\left(C_{a}\right) \text { for all } 1 \leq p, q \leq \infty ;
$$

see [4, 17, 22, 23] and the references therein for the discussion on spectrum of various convolution operators. Applying the above criterion again, together with the classical Wiener's lemma ([29]), it follows that the inverse of an $\ell^{p}$-stable convolution operator $C_{a}$ is a convolution operator $C_{b}$ associated with another summable sequence $b$. 


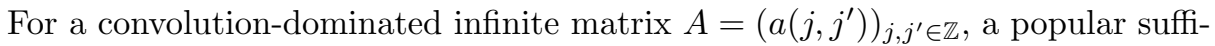
cient condition for its $\ell^{1}$-stability and $\ell^{\infty}$-stability is that $A$ is diagonal-dominated, i.e.,

$$
\inf _{j \in \mathbb{Z}}\left(|a(j, j)|-\max \left(\sum_{j^{\prime} \neq j}\left|a\left(j, j^{\prime}\right)\right|, \sum_{j^{\prime} \neq j}\left|a\left(j^{\prime}, j\right)\right|\right)\right)>0 .
$$

In this paper, we provide a practical criterion for the $\ell^{p}$-stability of convolutiondominated infinite matrices. We show that a convolution-dominated infinite matrix $A$ has $\ell^{p}$-stability if and only if it has certain "diagonal-blocks-dominated" property (see Theorem 2.1 for the precise statement).

\section{MAIN THEOREM}

To state our criterion for the $\ell^{p}$-stability of convolution-dominated infinite matrices, we introduce two concepts. Given an infinite matrix $A$, define the truncation matrices $A_{s}, s \geq 0$, by

$$
A_{s}=\left(a(i, j) \chi_{(-s, s)}(i-j)\right)_{i, j \in \mathbb{Z}},
$$

where $\chi_{E}$ is the characteristic function on a set $E$. Given $y \in \mathbb{R}$ and $1 \leq N \in \mathbb{Z}$, define the operator $\chi_{y}^{N}$ on $\ell^{p}$ by

$$
\chi_{y}^{N}: \ell^{p} \ni(c(j))_{j \in \mathbb{Z}} \longmapsto\left(c(j) \chi_{(-N, N)}(j-y)\right)_{j \in \mathbb{Z}} \in \ell^{p} .
$$

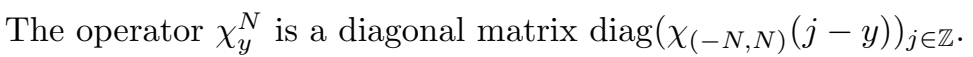

Theorem 2.1. Let $1 \leq p \leq \infty$, and let $A$ be a convolution-dominated infinite matrix in the class $\mathcal{C}$. Then the following statements are equivalent:

(i) The infinite matrix $A$ has $\ell^{p}$-stability.

(ii) There exist a positive constant $C_{0}$ and a positive integer $N_{0}$ such that

$$
\left\|\chi_{n}^{2 N} A \chi_{n}^{N} c\right\|_{p} \geq C_{0}\left\|\chi_{n}^{N} c\right\|_{p}, \quad c \in \ell^{p},
$$

hold for all integers $N \geq N_{0}$ and $n \in N \mathbb{Z}$.

(iii) There exist a positive integer $N_{0}$ and a positive constant $\alpha$ satisfying

$$
\alpha>2\left(5+2^{1-p}\right)^{1 / p} \inf _{0 \leq s \leq N_{0}}\left(\left\|A-A_{s}\right\|_{\mathcal{C}}+\frac{s}{N_{0}}\|A\|_{\mathcal{C}}\right)
$$

such that

$$
\begin{aligned}
& \qquad\left\|\chi_{n}^{2 N_{0}} A \chi_{n}^{N_{0}} c\right\|_{p} \geq \alpha\left\|\chi_{n}^{N_{0}} c\right\|_{p}, \quad c \in \ell^{p}, \\
& \text { hold for all } n \in N_{0} \mathbb{Z} \text {. }
\end{aligned}
$$

Taking $N_{0}=1$ in (2.2) and (2.3), we obtain a sufficient condition (2.4), which is a strong version of the diagonal-domination condition (1.7), for the $\ell^{\infty}$-stability of a convolution-dominated infinite matrix.

Corollary 2.2. Let $A=\left(a\left(j, j^{\prime}\right)\right)_{j, j^{\prime} \in \mathbb{Z}}$ be a convolution-dominated infinite matrix in the class $\mathcal{C}$. If

$$
\inf _{j \in \mathbb{Z}}\left(|a(j, j)|-2 \sum_{0 \neq k \in \mathbb{Z}} \sup _{j-j^{\prime}=k}\left|a\left(j, j^{\prime}\right)\right|\right)>0,
$$

then $A$ has $\ell^{\infty}$-stability. 
We say that an infinite matrix $A=(a(i, j))_{i, j \in \mathbb{Z}}$ is a band matrix if $a(i, j)=0$ for all $i, j \in \mathbb{Z}$ satisfying $j>i+k$ or $j<i-k$. The quantity $2 k+1$ is the bandwidth of the matrix $A$. For a band matrix $A$ with bandwidth $2 k+1, A-A_{s}$ is the zero matrix if $s>k$. Therefore for $N>k$,

$$
\inf _{0 \leq s \leq N}\left(\left\|A-A_{s}\right\|_{\mathcal{C}}+\frac{s}{N}\|A\|_{\mathcal{C}}\right) \leq \frac{k}{N}\|A\|_{\mathcal{C}} .
$$

This, together with Theorem 2.1. gives the following sufficient condition for a band matrix to have $\ell^{p}$-stability.

Corollary 2.3. Let $1 \leq p \leq \infty$, and let $A$ be a convolution-dominated band matrix in the class $\mathcal{C}$ with bandwidth $2 k+1$. If there exists an integer $N_{0}>k$ such that

$$
\left\|A \chi_{n}^{N_{0}} c\right\|_{p} \geq \alpha\left\|\chi_{n}^{N_{0}} c\right\|_{p}, \quad c \in \ell^{p},
$$

holds for some constant $\alpha$ strictly larger than $2\left(5+2^{1-p}\right)^{1 / p} k\|A\|_{\mathcal{C}} / N_{0}$, then $A$ has $\ell^{p}$-stability.

If we further assume that the infinite matrix $A$ in Corollary 2.3 has the form $A=\left(a\left(j-j^{\prime}\right)\right)_{j, j^{\prime} \in \mathbb{Z}}$ for some finite sequence $a=(a(j))_{j \in \mathbb{Z}}$ satisfying $a(j)=0$ for $|j|>k$, then $\|A\|_{\mathcal{C}}=\sum_{|j| \leq k}|a(j)|$ and the condition (2.5) can reformulated as follows:

$$
\left\|\tilde{A}_{N_{0}} c\right\|_{p} \geq \frac{\gamma k}{N_{0}}\left(\sum_{|j| \leq k}|a(j)|\right)\|c\|_{p}, \quad c \in \mathbb{R}^{2 N_{0}+1},
$$

holds for some $\gamma>2\left(5+2^{1-p}\right)^{1 / p}$, where

$$
\tilde{A}_{N_{0}}=\left(a\left(j-j^{\prime}\right)\right)_{-N_{0}-k \leq j \leq N_{0}+k,-N_{0} \leq j^{\prime} \leq N_{0}}
$$

and

$$
\|c\|_{p}= \begin{cases}\left(\sum_{j=-k_{1}}^{k_{2}}|c(j)|^{p}\right)^{1 / p} & \text { if } 1 \leq p<\infty \\ \sup _{-k_{1} \leq j \leq k_{2}}|c(j)| & \text { if } p=\infty\end{cases}
$$

for $c=\left(c\left(-k_{1}\right), \cdots, c(0), \ldots, c\left(k_{2}\right)\right)^{T} \in \mathbb{R}^{k_{1}+k_{2}+1}$. As a conclusion from (2.6) and (2.7), we see that if $A=\left(a\left(j-j^{\prime}\right)\right)_{j, j^{\prime} \in \mathbb{Z}}$ does not have $\ell^{p}$-stability, then for any large integer $N$,

$$
\inf _{0 \neq c \in \mathbb{R}^{2 N+1}} \frac{\left\|\tilde{A}_{N} c\right\|_{p}}{\|c\|_{p}} \leq \frac{2\left(5+2^{1-p}\right)^{1 / p} k}{N}\left(\sum_{|j| \leq k}|a(j)|\right) .
$$

For the special case $p=2$, the above inequality (2.8) can be interpreted as the minimal eigenvalue of $\left(\tilde{A}_{N}\right)^{T} \tilde{A}_{N}$ is less than or equal to $\frac{\sqrt{22} k^{2}}{N^{2}}\left(\sum_{|j| \leq k}|a(j)|\right)^{2}$, and it can also be rewritten as

$$
\inf _{0 \neq P_{N} \in \Pi_{N}} \frac{\left(\int_{-\pi}^{\pi}|\hat{a}(\xi)|^{2}\left|P_{N}(\xi)\right|^{2} d \xi\right)^{1 / 2}}{\left(\int_{-\pi}^{\pi}\left|P_{N}(\xi)\right|^{2} d \xi\right)^{1 / 2}} \leq \frac{\sqrt{22} k}{N}\left(\sum_{|j| \leq k}|a(j)|\right),
$$

where $\hat{a}(\xi)=\sum_{j \in \mathbb{Z}} a(j) e^{-i j \xi}$ and $\Pi_{N}$ is the set of all trigonometrical polynomials of degree at most $N$.

If the sequence $a=(a(j))_{j \in \mathbb{Z}}$ satisfies $a(0)=1, a(-1)=-1$, and $a(j)=0$ otherwise, then the bandwidth of the infinite matrix $A=\left(a\left(j-j^{\prime}\right)\right)_{j, j^{\prime} \in \mathbb{Z}}$ is equal to 3 , the norm $\|A\|_{\mathcal{C}}$ of the associated infinite matrix $A$ is equal to 2 , 


$$
\tilde{A}_{N}=\left(\begin{array}{cccccc}
-1 & 0 & 0 & \cdots & 0 & 0 \\
1 & -1 & 0 & \cdots & 0 & 0 \\
0 & 1 & -1 & \cdots & 0 & 0 \\
\vdots & \vdots & \ddots & \ddots & \vdots & \vdots \\
\vdots & \vdots & \ddots & \ddots & \ddots & \vdots \\
0 & 0 & 0 & \cdots & 1 & -1 \\
0 & 0 & 0 & \cdots & 0 & 1
\end{array}\right)
$$

and

$$
\inf _{0 \neq c \in \mathbb{R}^{2 N+1}} \frac{\left\|\tilde{A}_{N} c\right\|_{p}}{\|c\|_{p}} \geq \frac{1}{N+1},
$$

where the last inequality holds since the matrix

$$
\tilde{B}_{N}:=\left(\begin{array}{ccccccccccc}
-1 & 0 & 0 & \cdots & 0 & 0 & 0 & 0 & \cdots & 0 & 0 \\
-1 & -1 & 0 & \cdots & 0 & 0 & 0 & 0 & \cdots & 0 & 0 \\
-1 & -1 & -1 & \cdots & 0 & 0 & 0 & 0 & \cdots & 0 & 0 \\
\vdots & \vdots & \vdots & \ddots & \vdots & \vdots & \vdots & \vdots & \ddots & \vdots & \vdots \\
-1 & -1 & -1 & \cdots & -1 & 0 & 0 & 0 & \cdots & 0 & 0 \\
0 & 0 & 0 & \cdots & 0 & 0 & 1 & 1 & \cdots & 1 & 1 \\
0 & 0 & 0 & \cdots & 0 & 0 & 0 & 1 & \cdots & 1 & 1 \\
\vdots & \vdots & \vdots & \ddots & \vdots & \vdots & \vdots & \vdots & \ddots & \vdots & \vdots \\
0 & 0 & 0 & \cdots & 0 & 0 & 0 & 0 & \cdots & 1 & 1 \\
0 & 0 & 0 & \cdots & 0 & 0 & 0 & 0 & \cdots & 0 & 1
\end{array}\right)
$$

is a left inverse of the matrix $\tilde{A}_{N}$. Therefore the order $N^{-1}$ in (2.8) cannot be improved in general, but the author believes that the bound constant $2\left(5+2^{1-p}\right)^{1 / p}$ in (2.2) and (2.8) is not optimal and could be improved.

\section{Proof}

We say that a discrete subset $\Lambda$ of $\mathbb{R}^{d}$ is relatively-separated if

$$
R(\Lambda):=\sup _{x \in \mathbb{R}^{d}} \sum_{\lambda \in \Lambda} \chi_{\lambda+[-1 / 2,1 / 2)^{d}}(x)<\infty
$$

([1, 23, 27]). Clearly, the set $\mathbb{Z}$ of all integers is a relatively-separated subset of $\mathbb{R}$ with

$$
R(\mathbb{Z})=1
$$

Given a discrete set $\Lambda$, let $\ell^{p}(\Lambda)$ be the set of all $p$-summable sequences on the set $\Lambda$ with standard norm $\|\cdot\|_{\ell^{p}(\Lambda)}$ or $\|\cdot\|_{p}$ for brevity.

Given two relatively-separated subsets $\Lambda$ and $\Lambda^{\prime}$ of $\mathbb{R}^{d}$, define

$$
\mathcal{C}\left(\Lambda, \Lambda^{\prime}\right)=\left\{A:=\left(a\left(\lambda, \lambda^{\prime}\right)\right)_{\lambda \in \Lambda, \lambda^{\prime} \in \Lambda^{\prime}} \mid\|A\|_{\mathcal{C}\left(\Lambda, \Lambda^{\prime}\right)}<\infty\right\}
$$

where

$$
\|A\|_{\mathcal{C}\left(\Lambda, \Lambda^{\prime}\right)}=\sum_{k \in \mathbb{Z}^{d}} \sup _{\lambda \in \Lambda, \lambda^{\prime} \in \Lambda^{\prime}}\left|a\left(\lambda, \lambda^{\prime}\right)\right| \chi_{k+[-1 / 2,1 / 2]^{d}}\left(\lambda-\lambda^{\prime}\right) .
$$

It is obvious that

$$
\mathcal{C}(\mathbb{Z}, \mathbb{Z})=\mathcal{C}
$$


Given an infinite matrix $A=\left(a\left(\lambda, \lambda^{\prime}\right)\right)_{\lambda \in \Lambda, \lambda^{\prime} \in \Lambda^{\prime}}$, define its truncation matrices $A_{s}, s \geq 0$, by

$$
A_{s}=\left(a\left(\lambda, \lambda^{\prime}\right) \chi_{(-s, s)^{d}}\left(\lambda-\lambda^{\prime}\right)\right)_{\lambda \in \Lambda, \lambda^{\prime} \in \Lambda^{\prime}} .
$$

For any $y \in \mathbb{R}^{d}$ and a positive integer $N$, define the operator $\chi_{y}^{N}$ on $\ell^{p}(\Lambda)$ by

$$
\chi_{n}^{N}: \ell^{p}(\Lambda) \ni(c(\lambda))_{\lambda \in \Lambda} \longmapsto\left(c(\lambda) \chi_{(-N, N)^{d}}(\lambda-y)\right)_{\lambda \in \Lambda} \in \ell^{p}(\Lambda) .
$$

In this section, we establish the following criterion for the $\ell^{p}$-stability of infinite matrices in the class $\mathcal{C}\left(\Lambda, \Lambda^{\prime}\right)$, which is a slight generalization of Theorem 2.1 by (3.2) and (3.3).

Theorem 3.1. Let $1 \leq p \leq \infty$, the subsets $\Lambda, \Lambda^{\prime}$ of $\mathbb{R}^{d}$ be relatively-separated, and the infinite matrix $A$ belong to $\mathcal{C}\left(\Lambda, \Lambda^{\prime}\right)$. Then the following statements are equivalent to each other:

(i) The infinite matrix $A$ has $\ell^{p}$-stability, i.e., there exist positive constants $C_{1}$ and $C_{2}$ such that

(ii) There exist a positive constant $C_{0}$ and a positive integer $N_{0}$ such that

$$
\left\|\chi_{n}^{2 N} A \chi_{n}^{N} c\right\|_{\ell^{p}(\Lambda)} \geq C_{0}\left\|\chi_{n}^{N} c\right\|_{\ell^{p}\left(\Lambda^{\prime}\right)} \text { for all } c \in \ell^{p}\left(\Lambda^{\prime}\right),
$$

where $N_{0} \leq N \in \mathbb{Z}$ and $n \in N \mathbb{Z}^{d}$.

$\alpha>2\left(5+2^{1-p}\right)^{d / p} R(\Lambda)^{1 / p} R\left(\Lambda^{\prime}\right)^{1-1 / p} \inf _{0 \leq s \leq N_{0}}\left(\left\|A-A_{s}\right\|_{\mathcal{C}\left(\Lambda, \Lambda^{\prime}\right)}+\frac{d s}{N_{0}}\|A\|_{\mathcal{C}\left(\Lambda, \Lambda^{\prime}\right)}\right)$

such that

$$
\left\|\chi_{n}^{2 N_{0}} A \chi_{n}^{N_{0}} c\right\|_{\ell^{p}(\Lambda)} \geq \alpha\left\|\chi_{n}^{N_{0}} c\right\|_{\ell^{p}\left(\Lambda^{\prime}\right)}
$$

hold for all $c \in \ell^{p}\left(\Lambda^{\prime}\right)$ and $n \in N_{0} \mathbb{Z}$.

Using the above theorem, we obtain the following equivalence of $\ell^{p}$-stability for infinite matrices having certain off-diagonal decay, which is established in [2, 28, 23] for $\gamma>d(d+1), \gamma>0$, and $\gamma \geq 0$ respectively.

Corollary 3.2. Let $\Lambda, \Lambda^{\prime}$ be relatively-separated subsets of $\mathbb{R}^{d}$, and let $A=$ $\left(a\left(\lambda, \lambda^{\prime}\right)\right)_{\lambda \in \Lambda, \lambda^{\prime} \in \Lambda^{\prime}}$ satisfy

$$
\|A\|_{\mathcal{C}_{\gamma}\left(\Lambda, \Lambda^{\prime}\right)}=\sum_{k \in \mathbb{Z}^{d}}(1+|k|)^{\gamma} \sup _{\lambda \in \Lambda, \lambda^{\prime} \in \Lambda^{\prime}}\left|a\left(\lambda, \lambda^{\prime}\right)\right| \chi_{k+[-1 / 2,1 / 2]^{d}}\left(\lambda-\lambda^{\prime}\right)<\infty,
$$

where $\gamma>0$. Then the $\ell^{p}$-stability of the infinite matrix $A$ are equivalent to each other for different $1 \leq p \leq \infty$.

Proof. Let $1 \leq p \leq \infty$ and let $A$ have $\ell^{p}$-stability. Then by Theorem 3.1 there exists a positive constant $C_{0}$ and a positive integer $N_{0}$ such that

$$
\left\|\chi_{n}^{2 N} A \chi_{n}^{N} c\right\|_{\ell^{p}(\Lambda)} \geq C_{0}\left\|\chi_{n}^{N} c\right\|_{\ell^{p}\left(\Lambda^{\prime}\right)} \text { for all } c \in \ell^{p}\left(\Lambda^{\prime}\right),
$$

where $N_{0} \leq N \in \mathbb{Z}$ and $n \in N \mathbb{Z}^{d}$. From the equivalence of different norms on a finite-dimensional space, we have that

$$
\begin{aligned}
& \left((2 N)^{d} R(\Lambda)\right)^{\min (1 / q-1 / p, 0)}\left\|\chi_{n}^{N} c\right\|_{\ell^{p}(\Lambda)} \leq\left\|\chi_{n}^{N} c\right\|_{\ell^{q}(\Lambda)} \\
& \quad \leq\left((2 N)^{d} R(\Lambda)\right)^{\max (1 / q-1 / p, 0)}\left\|\chi_{n}^{N} c\right\|_{\ell^{p}(\Lambda)} \text { for all } c \in \ell^{p}(\Lambda),
\end{aligned}
$$


where $1 \leq p, q \leq \infty, 1 \leq N \in \mathbb{Z}$ and $n \in N \mathbb{Z}^{d}([2,23])$. Therefore for $1 \leq q \leq \infty$,

$$
\left\|\chi_{n}^{2 N} A \chi_{n}^{N} c\right\|_{\ell^{q}(\Lambda)} \geq C_{0}(2 N)^{-d|1 / p-1 / q|} R\left(\Lambda^{\prime}\right)^{\min (1 / p-1 / q, 0)}
$$

where $N_{0} \leq N \in \mathbb{Z}$ and $n \in N \mathbb{Z}^{d}$. We notice that

$$
\begin{aligned}
\inf _{0 \leq s \leq N}\left(\left\|A-A_{s}\right\|_{\mathcal{C}\left(\Lambda, \Lambda^{\prime}\right)}+\frac{d s}{N}\|A\|_{\mathcal{C}\left(\Lambda, \Lambda^{\prime}\right)}\right) & \leq\|A\|_{\mathcal{C}_{\gamma}\left(\Lambda, \Lambda^{\prime}\right)} \inf _{0 \leq s \leq N}\left(s^{\gamma}+\frac{d s}{N}\right) \\
& \leq(d+1)\|A\|_{\mathcal{C}_{\gamma}\left(\Lambda, \Lambda^{\prime}\right)} N^{-\gamma /(1+\gamma)} .
\end{aligned}
$$

Thus for $1 \leq q \leq \infty$ with $d|1 / p-1 / q|<\gamma /(1+\gamma)$, it follows from (3.10) and (3.11) that there exists a sufficiently large integer $N_{0}$ such that

$$
\left\|\chi_{n}^{2 N} A \chi_{n}^{N} c\right\|_{\ell^{q}(\Lambda)} \geq \alpha\left\|\chi_{n}^{N} c\right\|_{\ell^{q}\left(\Lambda^{\prime}\right)}
$$

hold for all $c \in \ell^{q}\left(\Lambda^{\prime}\right), N \geq N_{0}$ and $n \in N \mathbb{Z}^{d}$, where $\alpha$ is a positive constant larger than $2\left(5+2^{1-q}\right)^{d / q} R(\Lambda)^{1 / q} R\left(\Lambda^{\prime}\right)^{1-1 / q} \inf _{0 \leq s \leq N_{0}}\left(\left\|A-A_{s}\right\|_{\mathcal{C}\left(\Lambda, \Lambda^{\prime}\right)}+\frac{d s}{N_{0}}\|A\|_{\mathcal{C}\left(\Lambda, \Lambda^{\prime}\right)}\right)$. Then by Theorem 3.1, the infinite matrix $A$ has $\ell^{q}$-stability for all $1 \leq q \leq \infty$ with $d|1 / q-1 / p|<\gamma /(1+\gamma)$. Applying the above trick repeatedly, we prove the $\ell^{q}$-stability of the infinite matrix $A$ for any $1 \leq q \leq \infty$.

To prove Theorem 3.1, we first recall some basic properties for infinite matrices $A$ in the class $\mathcal{C}\left(\Lambda, \Lambda^{\prime}\right)$ and its truncation matrices $A_{s}, s \geq 0$.

Lemma 3.3 (23). Let $1 \leq p \leq \infty$, the subsets $\Lambda, \Lambda^{\prime}$ of $\mathbb{R}^{d}$ be relatively-separated, $A$ be an infinite matrix in the class $\mathcal{C}\left(\Lambda, \Lambda^{\prime}\right)$, and $A_{s}, s \geq 0$, be the truncation matrices of $A$. Then

$$
\begin{gathered}
\|A c\|_{\ell^{p}(\Lambda)} \leq R(\Lambda)^{1 / p} R\left(\Lambda^{\prime}\right)^{1-1 / p}\|A\|_{\mathcal{C}\left(\Lambda, \Lambda^{\prime}\right)}\|c\|_{\ell^{p}\left(\Lambda^{\prime}\right)} \quad \text { for all } c \in \ell^{p}\left(\Lambda^{\prime}\right), \\
\lim _{s \rightarrow+\infty}\left\|A-A_{s}\right\|_{\mathcal{C}\left(\Lambda, \Lambda^{\prime}\right)}=0 \\
\lim _{N \rightarrow} \inf _{+\infty}\left(\left\|A-A_{s}\right\|_{\mathcal{C}\left(\Lambda, \Lambda^{\prime}\right)}+\frac{d s}{N}\|A\|_{\mathcal{C}\left(\Lambda, \Lambda^{\prime}\right)}\right)=0
\end{gathered}
$$

and

$$
\left\|A_{s}\right\|_{\mathcal{C}} \leq\|A\|_{\mathcal{C}} \quad \text { for all } s \geq 0
$$

Let $\psi_{0}\left(x_{1}, \ldots, x_{d}\right)=\prod_{i=1}^{d} \max \left(\min \left(2-2\left|x_{i}\right|, 1\right), 0\right)$ be a cut-off function on $\mathbb{R}^{d}$. Then

$$
0 \leq \chi_{[-1 / 2,1 / 2]^{d}}(x) \leq \psi_{0}(x) \leq \chi_{(-1,1)^{d}}(x) \leq 1 \quad \text { for all } x \in \mathbb{R}^{d},
$$

and

$$
\left|\psi_{0}(x)-\psi_{0}(y)\right| \leq 2 d\|x-y\|_{\infty} \quad \text { for all } x, y \in \mathbb{R},
$$

where $\|x\|_{\infty}=\max _{1 \leq i \leq d}\left|x_{i}\right|$ for $x=\left(x_{1}, \ldots, x_{d}\right)$. Define the multiplication operator $\Psi_{n}^{N}$ on $\ell^{p}(\Lambda)$ by

$$
\Psi_{n}^{N}: \ell^{p}(\Lambda) \ni(c(\lambda))_{\lambda \in \Lambda} \longmapsto\left(\psi_{0}\left(\frac{\lambda-n}{N}\right) c(\lambda)\right)_{\lambda \in \Lambda} \in \ell^{p}(\Lambda) .
$$

Applying (3.17) and (3.18) for the cut-off function $\psi_{0}$, we obtain the following properties for the multiplication operators $\Psi_{n}^{N}, n \in N \mathbb{Z}$. 
Lemma 3.4. Let $1 \leq N \in \mathbb{Z}, \Lambda$ be a relatively-separated subset of $\mathbb{R}^{d}$, and the multiplication operators $\Psi_{n}^{N}, n \in N \mathbb{Z}^{d}$, be as in (3.19). Then

$$
\left\|\Psi_{n}^{N} c\right\|_{\ell^{p}(\Lambda)} \leq\left\|\chi_{n}^{N} c\right\|_{\ell^{p}(\Lambda)} \quad \text { for all } c \in \ell^{p}(\Lambda)
$$

where $1 \leq p \leq \infty$,

$$
\|c\|_{\ell^{p}(\Lambda)} \leq\left(\sum_{n \in N \mathbb{Z}^{d}}\left\|\Psi_{n}^{N} c\right\|_{\ell^{p}(\Lambda)}^{p}\right)^{1 / p} \leq 2^{d / p}\|c\|_{\ell^{p}(\Lambda)} \quad \text { for all } c \in \ell^{p}(\Lambda),
$$

$4^{d / p}\|c\|_{\ell^{p}(\Lambda)} \leq\left(\sum_{n \in N \mathbb{Z}^{d}}\left\|\Psi_{n}^{4 N} c\right\|_{\ell^{p}(\Lambda)}^{p}\right)^{1 / p} \leq\left(5+2^{1-p}\right)^{d / p}\|c\|_{\ell^{p}(\Lambda)} \quad$ for all $c \in \ell^{p}(\Lambda)$, where $1 \leq p<\infty$, and

$$
\|c\|_{\ell^{\infty}(\Lambda)}=\sup _{n \in N \mathbb{Z}^{d}}\left\|\Psi_{n}^{N} c\right\|_{\ell^{\infty}(\Lambda)}=\sup _{n \in N \mathbb{Z}^{d}}\left\|\Psi_{n}^{4 N} c\right\|_{\ell^{\infty}(\Lambda)} \quad \text { for all } c \in \ell^{\infty}(\Lambda) .
$$

To prove Theorem 2.1, we also need the following result.

Lemma 3.5 (23]). Let $N \geq 1$, the subsets $\Lambda, \Lambda^{\prime}$ of $\mathbb{R}^{d}$ be relatively-separated, $A$ be an infinite matrix in the class $\mathcal{C}\left(\Lambda, \Lambda^{\prime}\right), A_{N}$ be the truncation matrix of $A$, and $\Psi_{n}^{N}, n \in N \mathbb{Z}^{d}$, be the multiplication operators in (3.19). Then

$$
\left\|\Psi_{n}^{N} A_{N}-A_{N} \Psi_{n}^{N}\right\|_{\mathcal{C}\left(\Lambda, \Lambda^{\prime}\right)} \leq \inf _{0 \leq s \leq N}\left(\left\|A_{N}-A_{s}\right\|_{\mathcal{C}\left(\Lambda, \Lambda^{\prime}\right)}+\frac{2 d s}{N}\left\|A_{s}\right\|_{\mathcal{C}\left(\Lambda, \Lambda^{\prime}\right)}\right) .
$$

Now we start to prove Theorem 3.1 .

Proof of Theorem 3.1. (i) $\Longrightarrow$ (ii): By the $\ell^{p}$-stability of the infinite matrix $A$, there exists a positive constant $C_{0}$ (independent of $n \in N \mathbb{Z}^{d}$ and $1 \leq N \in \mathbb{Z}$ ) such that

$$
\left\|A \chi_{n}^{N} c\right\|_{\ell^{p}(\Lambda)} \geq C_{0}\left\|\chi_{n}^{N} c\right\|_{\ell^{p}\left(\Lambda^{\prime}\right)} \quad \text { for all } c \in \ell^{p}\left(\Lambda^{\prime}\right),
$$

where $n \in N \mathbb{Z}^{d}$ and $N \geq 1$. Noting that

$$
\chi_{n}^{2 N} A_{N} \psi_{n}^{N}=A_{N} \psi_{n}^{N}
$$

and applying (3.13) yield

$$
\begin{aligned}
& \left\|A \chi_{n}^{N} c-\chi_{n}^{2 N} A \chi_{n}^{N} c\right\|_{\ell^{p}(\Lambda)} \\
= & \left\|\left(I-\chi_{n}^{2 N}\right)\left(A-A_{N}\right) \chi_{n}^{N} c\right\|_{\ell^{p}(\Lambda)} \\
\leq & R(\Lambda)^{1 / p} R\left(\Lambda^{\prime}\right)^{1-1 / p}\left\|A-A_{N}\right\|_{\mathcal{C}\left(\Lambda, \Lambda^{\prime}\right)}\left\|\chi_{n}^{N} c\right\|_{\ell^{p}\left(\Lambda^{\prime}\right)},
\end{aligned}
$$

where $I$ is the identity operator. Combining the estimates in (3.25) and (3.27) proves that

$$
\left\|\chi_{n}^{2 N} A \chi_{n}^{N} c\right\|_{\ell^{p}(\Lambda)} \geq\left(C_{0}-R(\Lambda)^{1 / p} R\left(\Lambda^{\prime}\right)^{1-1 / p}\left\|A-A_{N}\right\|_{\mathcal{C}\left(\Lambda, \Lambda^{\prime}\right)}\right)\left\|\chi_{n}^{N} c\right\|_{\ell^{p}\left(\Lambda^{\prime}\right)}
$$

hold for all $c \in \ell^{p}\left(\Lambda^{\prime}\right)$, where $n \in N \mathbb{Z}^{d}$ and $N \geq 1$. The conclusion (ii) then follows from (3.14) and (3.28).

(ii) $\Longrightarrow$ (iii): The implication follows from (3.15).

(iii) $\Longrightarrow$ (i): $\quad$ Let $1 \leq p<\infty$. Take any $n \in N_{0} \mathbb{Z}^{d}$ and $c \in \ell^{p}\left(\Lambda^{\prime}\right)$. By the assumption (iii) for the infinite matrix $A$,

$$
\left\|\chi_{n}^{2 N_{0}} A \Psi_{n}^{N_{0}} c\right\|_{\ell^{p}(\Lambda)}=\left\|\chi_{n}^{2 N_{0}} A \chi_{n}^{N_{0}} \Psi_{n}^{N_{0}} c\right\|_{\ell^{p}(\Lambda)} \geq \alpha\left\|\Psi_{n}^{N_{0}} c\right\|_{\ell^{p}\left(\Lambda^{\prime}\right)} .
$$


This together with (3.13) and (3.26) implies that

$$
\begin{aligned}
& \left\|A_{N_{0}} \Psi_{n}^{N_{0}} c\right\|_{\ell^{p}(\Lambda)} \\
= & \left\|\chi_{n}^{2 N_{0}}\left(A_{N_{0}}-A+A\right) \Psi_{n}^{N_{0}} c\right\|_{\ell^{p}(\Lambda)} \\
\geq & \left\|\chi_{n}^{2 N_{0}} A \chi_{n}^{N_{0}} \Psi_{n}^{N_{0}} c\right\|_{\ell^{p}(\Lambda)}-\left\|\chi_{n}^{2 N_{0}}\left(A_{N_{0}}-A\right) \Psi_{n}^{N_{0}} c\right\|_{\ell^{p}(\Lambda)} \\
\geq & \left(\alpha-R(\Lambda)^{1 / p} R\left(\Lambda^{\prime}\right)^{1-1 / p}\left\|A-A_{N_{0}}\right\|_{\mathcal{C}\left(\Lambda, \Lambda^{\prime}\right)}\right)\left\|\Psi_{n}^{N_{0}} c\right\|_{\ell^{p}\left(\Lambda^{\prime}\right)} .
\end{aligned}
$$

From (3.13) and (3.24) it follows that

$$
\begin{aligned}
& \left\|\left(\Psi_{n}^{N_{0}} A_{N_{0}}-A_{N_{0}} \Psi_{n}^{N_{0}}\right) c\right\|_{\ell^{p}(\Lambda)} \\
= & \left\|\left(\Psi_{n}^{N_{0}} A_{N_{0}}-A_{N_{0}} \Psi_{n}^{N_{0}}\right) \Psi_{n}^{4 N_{0}} c\right\|_{\ell^{p}(\Lambda)} \\
\leq & R(\Lambda)^{1 / p} R\left(\Lambda^{\prime}\right)^{1-1 / p}\left\|\Psi_{n}^{N_{0}} A_{N_{0}}-A_{N_{0}} \Psi_{n}^{N_{0}}\right\|_{\mathcal{C}\left(\Lambda, \Lambda^{\prime}\right)}\left\|\Psi_{n}^{4 N_{0}} c\right\|_{\ell^{p}\left(\Lambda^{\prime}\right)} \\
\leq & R(\Lambda)^{1 / p} R\left(\Lambda^{\prime}\right)^{1-1 / p} \\
& \times \inf _{0 \leq s \leq N_{0}}\left(\left\|A_{N_{0}}-A_{s}\right\|_{\mathcal{C}}+\frac{2 d s}{N_{0}}\left\|A_{N_{0}}\right\|_{\mathcal{C}}\right)\left\|\Psi_{n}^{4 N_{0}} c\right\|_{\ell^{p}\left(\Lambda^{\prime}\right)} .
\end{aligned}
$$

Combining (3.21), (3.22), (3.30) and (3.31), we get

$$
\begin{aligned}
& 2^{d / p}\left\|A_{N_{0}} c\right\|_{\ell^{p}(\Lambda)} \geq\left(\sum_{n \in N_{0} \mathbb{Z}}\left\|\Psi_{n}^{N_{0}} A_{N_{0}} c\right\|_{\ell^{p}(\Lambda)}^{p}\right)^{1 / p} \\
\geq & \left(\alpha-R(\Lambda)^{1 / p} R\left(\Lambda^{\prime}\right)^{1-1 / p}\left\|A-A_{N_{0}}\right\|_{\mathcal{C}\left(\Lambda, \Lambda^{\prime}\right)}\right)\left(\sum_{n \in N_{0} \mathbb{Z}}\left\|\Psi_{n}^{N_{0}} c\right\|_{\ell^{p}\left(\Lambda^{\prime}\right)}^{p}\right)^{1 / p} \\
& -R(\Lambda)^{1 / p} R\left(\Lambda^{\prime}\right)^{1-1 / p} \inf _{0 \leq s \leq N_{0}}\left(\left\|A_{N_{0}}-A_{s}\right\|_{\mathcal{C}\left(\Lambda, \Lambda^{\prime}\right)}+\frac{2 d s}{N_{0}}\left\|A_{N_{0}}\right\|_{\mathcal{C}\left(\Lambda, \Lambda^{\prime}\right)}\right) \\
& \times\left(\sum_{n \in N_{0} \mathbb{Z}}\left\|\Psi_{n}^{4 N_{0}} c\right\|_{\ell^{p}\left(\Lambda^{\prime}\right)}^{p}\right)^{1 / p} \\
\geq & \left(\alpha-R(\Lambda)^{1 / p} R\left(\Lambda^{\prime}\right)^{1-1 / p}\left\|A-A_{N_{0}}\right\|_{\mathcal{C}\left(\Lambda, \Lambda^{\prime}\right)}-\left(5+2^{1-p}\right)^{1 / p} R(\Lambda)^{1 / p} R\left(\Lambda^{\prime}\right)^{1-1 / p}\right. \\
& \left.\times \inf _{0 \leq s \leq N_{0}}\left(\left\|A_{N_{0}}-A_{s}\right\|_{\mathcal{C}\left(\Lambda, \Lambda^{\prime}\right)}+\frac{2 d s}{N_{0}}\left\|A_{N_{0}}\right\|_{\mathcal{C}\left(\Lambda, \Lambda^{\prime}\right)}\right)\right)\|c\|_{\ell^{p}\left(\Lambda^{\prime}\right)} .
\end{aligned}
$$

Therefore

$$
\begin{aligned}
& \|A c\|_{\ell^{p}(\Lambda)} \geq\left\|A_{N_{0}} c\right\|_{\ell^{p}(\Lambda)}-\left\|\left(A-A_{N_{0}}\right) c\right\|_{\ell^{p}(\Lambda)} \\
\geq & 2^{-1 / p}\left(\alpha-\left(1+2^{d / p}\right) R(\Lambda)^{1 / p} R\left(\Lambda^{\prime}\right)^{1-1 / p}\left\|A-A_{N_{0}}\right\|_{\mathcal{C}\left(\Lambda, \Lambda^{\prime}\right)}\right. \\
& -\left(5+2^{1-p}\right)^{d / p} R(\Lambda)^{1 / p} R\left(\Lambda^{\prime}\right)^{1-1 / p} \\
& \left.\times \inf _{0 \leq s \leq N_{0}}\left(\left\|A_{N_{0}}-A_{s}\right\|_{\mathcal{C}\left(\Lambda, \Lambda^{\prime}\right)}+\frac{2 d s}{N_{0}}\left\|A_{N_{0}}\right\|_{\mathcal{C}\left(\Lambda, \Lambda^{\prime}\right)}\right)\right)\|c\|_{\ell^{p}\left(\Lambda^{\prime}\right)} \\
\geq & 2^{-d / p}\left(\alpha-2\left(5+2^{1-p}\right)^{1 / p} R(\Lambda)^{1 / p}\right. \\
& \left.\times R\left(\Lambda^{\prime}\right)^{1-1 / p} \inf _{0 \leq s \leq N_{0}}\left(\left\|A-A_{s}\right\|_{\mathcal{C}\left(\Lambda, \Lambda^{\prime}\right)}+\frac{d s}{N_{0}}\|A\|_{\mathcal{C}\left(\Lambda, \Lambda^{\prime}\right)}\right)\right)\|c\|_{\ell^{p}\left(\Lambda^{\prime}\right)},
\end{aligned}
$$

and the conclusion (i) for $1 \leq p<\infty$ follows.

The conclusion (i) for $p=\infty$ can be proved by a similar argument. We omit the details here. 


\section{ACKNOWLEDGMENTS}

The author thanks Professors Deguang Han, Zuhair M. Nashed, Xianliang Shi, and Wai-Shing Tang for their discussion and suggestions in preparing the manuscript.

\section{REFERENCES}

[1] A. Aldroubi and K. Gröchenig, Nonuniform sampling and reconstruction in shift-invariant space, SIAM Review, 43(2001), 585-620. MR.1882684 (2003e:94040)

[2] A. Aldroubi, A. Baskakov and I. Krishtal, Slanted matrices, Banach frames, and sampling, J. Funct. Anal., 255(2008), 1667-1691. MR2442078(2010a:46059)

[3] R. Balan, P. G. Casazza, C. Heil, and Z. Landau, Density, overcompleteness and localization of frames. I. Theory; II. Gabor systems, J. Fourier Anal. Appl., 12(2006), 105-143; 309-344. MR2224392 (2007b:42041) MR2235170 (2007b:42042)

[4] B. A. Barnes, When is the spectrum of a convolution operator on $L^{p}$ independent of $p$ ? Proc. Edinburgh Math. Soc., 33(1990), 327-332. MR.1057760(91f:47046)

[5] A. G. Baskakov, Wiener's theorem and asymptotic estimates for elements of inverse matrices, Funktsional. Anal. i Prilozhen., 24(1990), 64-65; translation in Funct. Anal. Appl., 24(1990), 222-224. MR 1082033 (92g:47049)

[6] C. K. Chui, W. He, and J. Stöckler, Nonstationary tight wavelet frames. I. Bounded intervals; II: Unbounded intervals, Appl. Comp. Harmonic Anal., 17(2004), 141-197; 18(2005), 25-66. MR2082157 (2005f:42082) MR2110512 (2005j:42026)

[7] A. Connes, $C^{*}$ algèbres et géométrie differentielle. C. R. Acad. Sci. Paris Sér. A-B, 290(1980), A599-A604. MR.572645 (81c:46053)

[8] C. de Boor, A bound on the $L_{\infty}$-norm of the $L_{2}$-approximation by splines in terms of a global mesh ratio, Math. Comp., 30(1976), 687-694. MR0425432 (54:13387)

[9] S. Demko, Inverse of band matrices and local convergences of spline projections, SIAM J. Numer. Anal., 14(1977), 616-619. MR0455281 (56:13520)

[10] G. Fendler, K. Gröchenig, and M. Leinert, Convolution-dominated operators on discrete groups, Integral Equations Operator Theory, 61(2008), 493-509. MR2434338(2009m:47085)

[11] I. M. Gelfand, D. A. Raikov, and G. E. Silov, Commutative Normed Rings, Chelsea, New York, 1964. MR0205105(34:4940)

[12] I. Gohberg, M. A. Kaashoek, and H. J. Woerdeman, The band method for positive and strictly contractive extension problems: An alternative version and new applications, Integral Equations Operator Theory, 12(1989), 343-382. MR998278 (90c:47022)

[13] K. Gröchenig, Time-frequency analysis of Sjöstrand's class, Rev. Mat. Iberoamer., 22(2006), 703-724. MR2294795 (2008b:35308)

[14] K. Gröchenig and M. Leinert, Wiener's lemma for twisted convolution and Gabor frames, J. Amer. Math. Soc., 17(2003), 1-18. MR2015328 (2004m:42037)

[15] K. Gröchenig and M. Leinert, Symmetry and inverse-closedness of matrix algebras and functional calculus for infinite matrices, Trans. Amer. Math. Soc., 358(2006), 2695-2711. MR2204052 (2006k:47065)

[16] K. Gröchenig and T. Strohmer, Pseudodifferential operators on locally compact abelian groups and Sjöstrand's symbol class, Journal für die reine und angewandte Mathematik, 613(2007), 121-146. MR2377132 (2009d:35360)

[17] A. Hulanicki, On the spectrum of convolution operators on groups with polynomial growth, Invent. Math., 17(1972), 135-142. MR0323951(48:2304)

[18] S. Jaffard, Properiétés des matrices bien localisées prés de leur diagonale et quelques applications, Ann. Inst. Henri Poincaré, 7(1990), 461-476. MR.1138533 (93h:47035)

[19] R.-Q. Jia and C. A. Micchelli, Using the refinement equations for the construction of prewavelets. II. Powers of two, in Curves and Surfaces (Chamonix-Mont-Blanc, 1990), Academic Press, Boston, MA, 1991, 209-246. MR1123739 (93e:65024)

[20] V. G. Kurbatov, Algebras of difference and integral operators, Funktsional. Anal. i Prilozhen., 24(1990), 87-88. MR1069415 (91k:47067)

[21] M. A. Naimark, Normed Algebras, Wolters-Noordhoff Publishing, Groningen, 1972. MR0438123(55:11042) 
[22] T. Pytlik, On the spectral radius of elements in group algrebras, Bull. Acad. Polon. Sci. Ser. Sci. Math., 21(1973), 899-902. MR0328476 (48:6818)

[23] C. E. Shin and Q. Sun, Stability of localized operators, J. Funct. Anal., 256(2009), 24172439. MR2502521 (2010e:47012)

[24] J. Sjöstrand, An algebra of pseudodifferential operators, Math. Res. Lett., 1(1994), 185-192. MR1266757 (95b:47065)

[25] J. Sjöstrand, Wiener type algebras of pseudodifferential operators, Centre de Mathematiques, École Polytechnique, Palaiseau, France, Séminaire 1994-1995, 1995. MR.1362552 (96j:47049)

[26] Q. Sun, Non-uniform sampling and reconstruction for signals with finite rate of innovations, SIAM J. Math. Anal., 38(2006), 1389-1422. MR2286012(2008b:94049)

[27] Q. Sun, Wiener's lemma for infinite matrices, Trans. Amer. Math. Soc., 359(2007), 30993123. MR.2299448 (2008e:47036)

[28] R. Tessera, Finding left inverse for operators on $\ell^{p}\left(\mathbb{Z}^{d}\right)$ with polynomial decay, preprint, 2008. arXiv:0801.1532

[29] N. Wiener, Tauberian theorems, Ann. of Math. (2), 33(1932), 1-100. MR.1503035

Department of Mathematics, University of Central Florida, Orlando, Florida 32816

E-mail address: qsun@mail.ucf.edu 